\title{
Long-Term Survival of Resected Pancreatic Carcinoma Which Was Coincidentally Detected at the Occurrence of Incarcerated Inguinal Hernia: A Case Report
}

\author{
Shigeru Fujisaki1 ${ }^{*}$, Motoi Takashina ${ }^{1}$, Ryouichi Tomita ${ }^{2}$, Ken-Ichi Sakurai ${ }^{3}$, \\ Tadatoshi Takayama ${ }^{4}$ \\ ${ }^{1}$ Department of Surgery, Fujisaki Hospital, Tokyo, Japan \\ ${ }^{2}$ Department of Surgery, Nippon Dental University, Tokyo, Japan \\ ${ }^{3}$ Division of Breast and Endocrine Surgery, Nihon University School of Medicine, Tokyo, Japan \\ ${ }^{4}$ Division of Digestive Surgery, Nihon University School of Medicine, Tokyo, Japan \\ Email: *sfujisak@blue.ocn.ne.jp
}

How to cite this paper: Fujisaki, S., Takashina, M., Tomita, R., Sakurai, K.-I. and Takayama, T. (2018) Long-Term Survival of Resected Pancreatic Carcinoma Which Was Coincidentally Detected at the Occurrence of Incarcerated Inguinal Hernia: A Case Report. Journal of Cancer Therapy, 9, 516-521.

https://doi.org/10.4236/jct.2018.96042

Received: May 29, 2018

Accepted: June 22, 2018

Published: June 25, 2018

Copyright (C) 2018 by authors and Scientific Research Publishing Inc. This work is licensed under the Creative Commons Attribution International License (CC BY 4.0).

http://creativecommons.org/licenses/by/4.0/

\begin{abstract}
Surgical intervention of asymptomatic and accidentally detected pancreatic carcinoma can prolong survival. A 67-year-old man with the right incarcerated inguinal hernia was referred to our hospital, he immediately underwent manipulative treatment followed by hernioplasty on the next day. Upon the first visit, a pancreatic tumor was accidentally detected in CT images in the pancreatic tail. About approximately a month, abdominal CT revealed a slightly developed tumor; accordingly, distal pancreatectomy with lymph node dissection was performed. The patient was histopathologically diagnosed with tubular adenocarcinoma, and his final pathological stage was ypT2, pN0, cM0, Stage Ib, based on the TNM classification of malignant tumors (8th edition). For postoperative six months, he was treated with adjuvant chemotherapy using gemcitabine $\left(1000 \mathrm{mg} / \mathrm{m}^{2}\right)$. Remarkably, the patient reported no recurrence and has been alive for postoperative 7.5 years, thereby attaining excellent outcomes for accidentally detected pancreatic carcinoma at the occurrence of an incarcerated inguinal hernia.
\end{abstract}

\section{Keywords}

Pancreatic Carcinoma, Incarcerated Inguinal Hernia, Long-Term Survival

\section{Introduction}

Pancreatic carcinoma has exhibits a poor prognosis with limited long-term sur- 
vival. Because pancreatic carcinoma displays minimal symptoms at an early stage, it is often diagnosed at an advanced stage when treatment options are scarce. Even if it is asymptomatic and an accidentally detected, its early detection is imperative to initiate the relevant treatment as soon as possible. To the best of our knowledge, no study has yet reported a case of asymptomatic pancreatic carcinoma that has been accidentally detected when the patient visited hospital at the occurrence of other abdominal diseases, resected, and still obtained excellent prognosis.

Herein, we report the case of a long-term survivor following curative resection for pancreatic ductal adenocarcinoma (PDAC) that was coincidentally detected at the occurrence of incarcerated inguinal hernia.

\section{Case Report}

In October 2010, a 67-year-old man with the right incarcerated inguinal hernia was referred to our hospital (Figure 1); he immediately underwent manipulative treatment followed by mesh plug hernioplasty the next day.

At the first visit, plain CT detected a pancreatic tumor (Figure 2), which was confirmed to be of $2.9 \mathrm{~cm}$ in diameter in the pancreatic tail by enhanced CT performed immediately after the hernia surgery (Figure 3). At that point, the patient did not display any pancreatic tumor-related symptoms and declined to undergo a close examination such as biopsy, thus, we inevitably decided to observe the patient for a month. After approximately a month of the initial examination, abdominal CT revealed the tumor to be slightly enlarged to $3.5 \mathrm{~cm}$ in diameter (Figure 4). However, compared with a month before, as cites retention, lymph node enlargement, and distant metastases were not detected. In December 2010, the patient underwent distal pancreatectomy with lymph node dissection (Figure 5). Notably, no metastatic lesion, as cites, or disseminated lesions were detected in the peritoneal cavity.

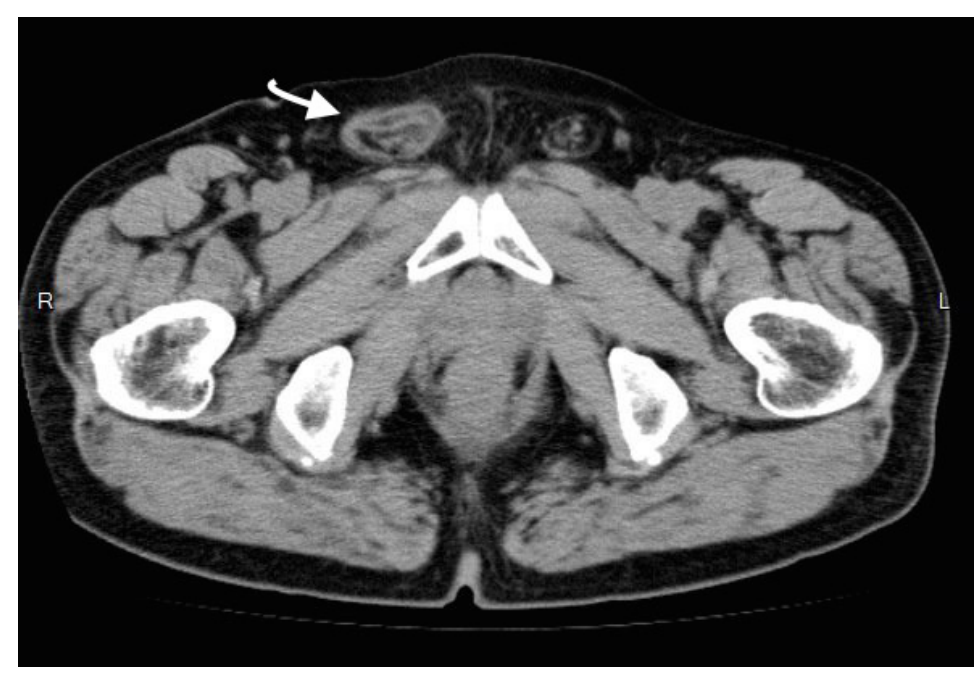

Figure 1. Plain abdominal computed tomography showing the right inguinal hernia with an extended sac (arrow). 


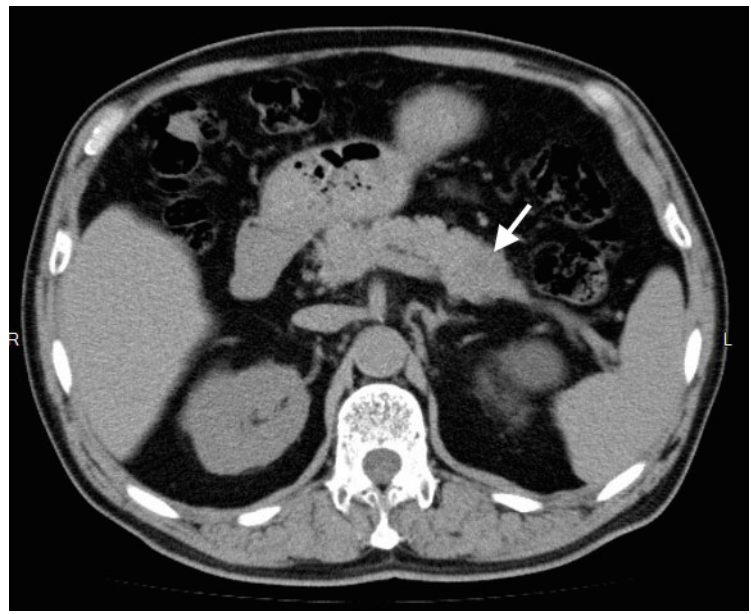

Figure 2. Plain abdominal computed tomography showing a low-density mass lesion in the pancreatic tail (arrow).

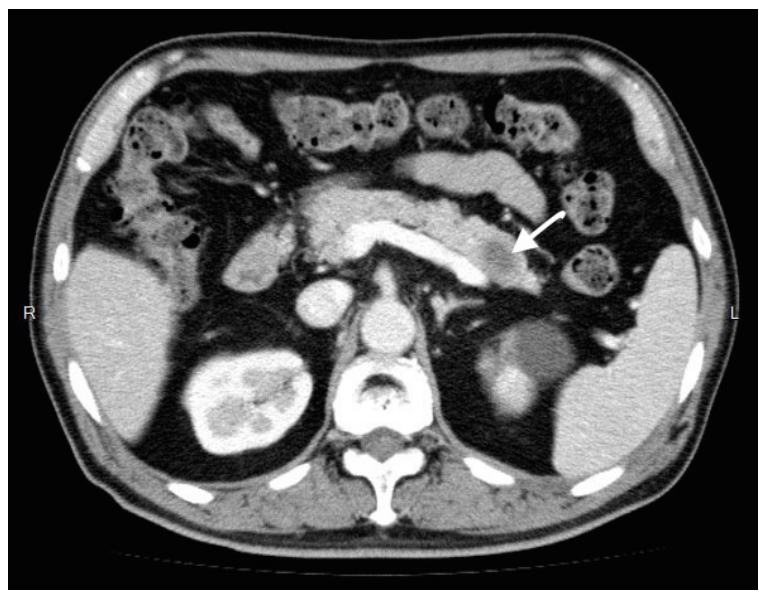

Figure 3. Enhanced abdominal computed tomography showing a pancreatic tumor with a diameter of $2.9 \mathrm{~cm}$ in the pancreatic tail. In the arterial phase, it was also of low density compared with pancreatic parenchyma and seemed to be a hypovascular tumor mass (arrow).

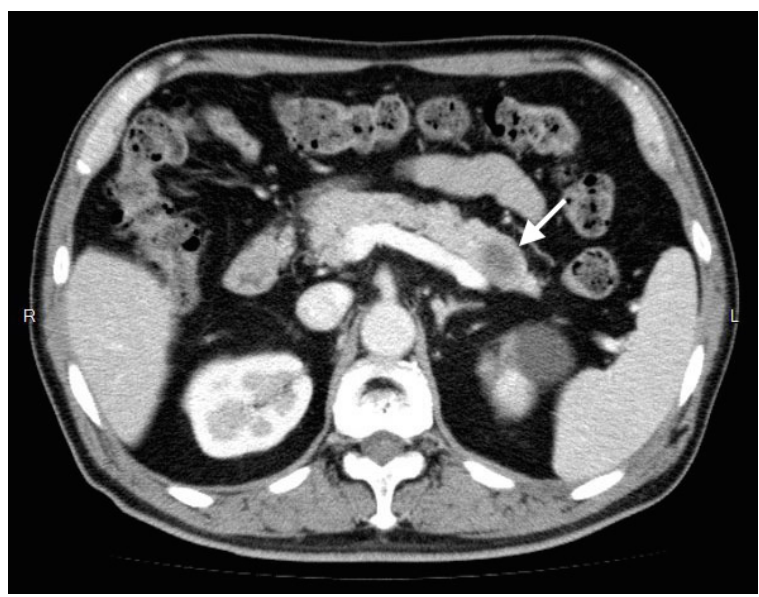

Figure 4. About a month from the initial CT, abdominal CT revealed that the tumor had slightly enlarged to $3.5 \mathrm{~cm}$ in diameter (arrow). 


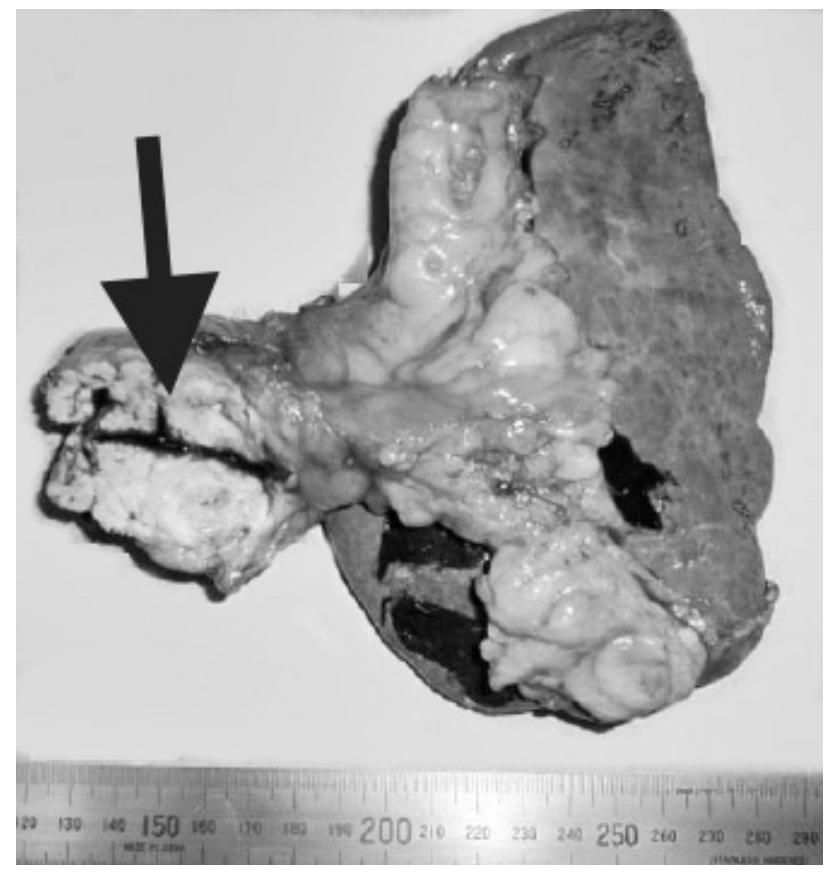

Figure 5. A surgical specimen, the tumor incised in the middle following surgery, was curatively resected (black arrow).

Histopathologically, the patient was diagnosed with tubular adenocarcinoma, and his final pathological stage was ypT2, pN0, cM0, stage Ib, based on the TNM classification of malignant tumors, 8th edition.

Later, he developed a grade A postoperative pancreatic fistula [1]. After postoperative 20 days, the drain was removed; the patient was discharged on postoperative 30 days. Further, on postoperative day 38, we initiated adjuvant chemotherapy comprising gemcitabine $\left(1000 \mathrm{mg} / \mathrm{m}^{2}\right)$ for six months. To date, the patient reported no recurrence and has been alive for postoperative 7 years and 6 months.

\section{Discussion}

As patients with pancreatic carcinoma display minimal symptoms at an early stage, it is often diagnosed at an advanced stage when treatment options are scarce. Therefore, early detection of pancreatic carcinoma, while symptoms are poor, it is the gospel for these patients.

Asymptomatic PDAC is associated with improved long-term outcomes than symptomatic PDAC because of the earlier stage at presentation and a higher probability of resectability [2]. In addition, establishing screening programs for the early detection of PDAC in selected high-risk populations is imperative. While it is crucial to attempt early detection of pancreatic carcinoma by focusing on high risk groups, it is also essential to focus on pancreatic lesions through every opportunity to perform abdominal imaging studies for other diseases. While examining the abdomen of patients, clinicians should also consider the possibility of the presence of pancreatic carcinoma. Among the patients who vi- 
sited due to acute abdomen, most of cases in which pancreatic cancer was detected have diseases that were directly related to pancreatic cancer, that is, diseases caused by concomitant pancreatitis or invasion and dissemination of pancreatic cancer [3] [4]. On the other hand, there have been no case reports like our case, in which pancreatic cancer was incidentally detected at the time of visit by other acute abdomen disease. In our case, the patient visited our hospital with an incarcerated inguinal hernia and a coexistence of pancreatic carcinoma, which was completely unrelated to his hernia. An accidental detection of a pancreatic tumor by CT led to curative treatment for the disease, ensuring the patient's prolonged survival.

A preoperative biopsy of pancreatic lesions suspected of malignancy is debatable. Hartwig $\mathrm{W}$ et al. illustrated that a preoperative biopsy of potentially resectable pancreatic tumors is not usually advisable, as malignancy cannot be eliminated with adequate reliability [5]. In our case, when a pancreatic lesion was detected, the patient declined to undergo post operative survival without recurrence for $>7$ years.

To the best of our knowledge, no report exists on whether a biopsy will be, apparently, harmful for obtaining long-term survival in patients with PDAC. Owing to a risk of implantation within the abdominal cavity during puncture and aspiration [6], it is estimated that a preoperative biopsy could be unsuitable for the long-term survival.

\section{Conclusion}

Because pancreatic carcinoma is associated with poor prognosis, the presence or absence of pancreatic tumor should be focused while performing abdominal imaging for the diagnosis of any abdominal diseases.

\section{Acknowledgements}

The authors declare that they have no competing interests.

\section{References}

[1] Pratt, W.B., Maithel, S.K., Vanounou, T., Huang, Z.S., Callery, M.P. and Vollmer Jr., C.M. (2007) Clinical and Economic Validation of the International Study Group of Pancreatic Fistula (ISGPF) Classification Scheme. Annals of Surgery, 245, 443-451. https://doi.org/10.1097/01.sla.0000251708.70219.d2

[2] Takeda, Y., Saiura, A., Takahashi, Y., Inoue, Y., Ishizawa, T., Mise, Y., Matsumura, M., Ichida, H., Matsuki, R., Tanaka, M. and Ito, H. (2017) Asymptomatic Pancreatic Cancer: Does Incidental Detection Impact Long-Term Outcomes? Journal of Gastrointestinal Surgery, 21, 1287-1295. https://doi.org/10.1007/s11605-017-3421-2

[3] Gambill, E.E. (1971) Pancreatitis Associated with Pancreatic Carcinoma: A Study of 26 Cases. Mayo Clinic Proceeding, 46, 174-177.

[4] Griffin, R., Villas, B., Davis, C. and Awad, Z.T. (2012) Carcinoma of the Tail of the Pancreas Presenting as Acute Abdomen. Journal of the Pancreas, 13, 58-60.

[5] Hartwig, W., Schneider, L., Diener, M.K., Bergmann, F., Büchler, M.W. and Werner, J. (2009) Preoperative Tissue Diagnosis for Tumours of the Pancreas. British 
Journal of Surgery, 96, 5-20. https://doi.org/10.1002/bjs.6407

[6] Yasumoto, M., Okabe, Y., Ishikawa, H., Kisaki, J., Akiba, J., Naito, Y., Ishida, Y., Ushijima, T., Tsuruta, O. and Torimura T.(2018) A Case of Gastric Wall Implantation Caused by EUS-FNA 22 Months after Pancreatic Cancer Resection. Endoscopic Ultrasound, 7, 64-66. 